\title{
Games and The Fluidity of Layered Agency
}

\section{Luca Ferrero}

Philosophy, University of California-Riverside, Riverside, CA, USA

luca.ferrero@ucr.edu

forthcoming in a special issue on of the Journal of the Philosophy of Sport

on C. Thi Nguyen 'Games: Agency as Art'

edited by Christopher Yorke and John Russell

PLEASE CITE or QUOTE from PUBLISHED VERSION ONCE AVAILABLE

Draft of 07 May 2021 


\section{Games and The Fluidity of Layered Agency}

Keywords: games; agency; fluidity

\section{Introduction}

What can the philosophy of agency learn from Nguyen's (2020a) book on games? The most important lesson concerns, to use Nguyen's terms, the 'layered' structure of our agency and the 'fluidity' required to establish and navigate this structure. These features of agency have not gone unnoticed but I believe that they have not been sufficiently theorized. In this paper, I will work through the implications of Nguyen's account of striving games to offer a preliminary sketch of a general account of fluid layered agency. I am entirely persuaded by Nguyen's account of the nature and operation of striving games, so I am offering this paper not in the spirit of criticism but rather of a celebration of his groundbreaking work. This paper is just a series of moves in a cooperative philosophical game.

\section{Flexible and Fully Transparent Agency}

To better understand the structure of fluid layered agency, let's begin by looking at flat, flexible, and fully transparent agency.

Consider an agent $A$ who is at the Salt Flats on the Bonneville Speedway in Utah. $A$ has been stranded on one of the tracks and she is trying to rejoin her teammates a few miles away. $A$ can see her team at a distance but she cannot rule out that the team might be moving at some point from their current location. So, as she tries to get closer to them, she needs to constantly check for their current position and adjust her trajectory accordingly.

The practical predicament of $A$ is straightforward. $A$ needs to calculate a path (hopefully an efficient one) that would take her from her present location to the visible current location of 
the team and take the first step in that direction. Because of the Speedway flatness, $A$ needs not to worry about making adjustments for the terrain ahead of her; she need not worry about vegetation, water, elevation, etc. That is why the best and easiest plan for her is to draw a straight line from her current location to the location of the team and move right away in that direction.

Nonetheless, this plan must stay maximally flexible. If the team were to move before $A$ reaches them, $A-$ who continues to have a clear view of their location-would immediately recalculate a new and better path and adjust her steps accordingly. In this scenario, $A$ has only one end: to rejoin the team. There are no intermediate ends. Although $A$ would have to achieve some milestones on the way to eventual success, such as taking the next step in that direction or halving the distance between her and the team, none of these milestones counts as a genuine end. These milestones do not perform any guiding role in her reasoning and action. They are rather automatically achieved as $A$ continues to make good progress along her chosen path.

$A$ 's agency is fully transparent and operates in a perfectly flat landscape. There is something ideal about this agency: the complete dependency of means to a final end, including their maximal instrumental flexibility. Within the range of available means, $A$ can immediately adopt them in response to any changes in the path to her final end.

We enjoy such flexibility for some of our actions, especially over short time ranges, such as extending our arm to grasp a moving and fully visible object or walking toward a friend on the street. In these cases, we focus directly on a continuously visible target and we are ready to make immediate instrumental adjustments to reach it.

Unfortunately, a fully transparent agency becomes impossible for more distant and complex goals in non-flat landscapes. What we get, instead, is a layered agency, as Nguyen aptly 
calls it. As I will explain below, the layering is a matter of segments, strata, and niches generated by the acquisition of genuine but only intermediate and potentially disposable ends, which we adopt and pursue on the way to our final ends. This agency is no longer maximally flexible: the intermediate ends set constraints on the paths and means to the final ends. But this agency is not completely rigid either. Rather, it is — to use again Nguyen's terminology—fluid. By 'fluidity' I mean the capacity both to set up, operate within, and remove layers (i.e., to adopt, pursue, and discard intermediate ends) and to navigate across them.

\section{Horizontal Segments}

Let's imagine that $A$ is now stranded farther away from her team, on the other side of Goshute Peak, without a direct view of her team and unable to gather that information by any other means. This new feature of her landscape forces a segmentation of her path to the final end. She needs to acquire a genuine intermediate end: to get around the peak. This is no longer simply a means to her final end. Given that she cannot see the location of her team until she gets around the peak, up to that time, it is physically impossible for $A$ knowingly to adjust her conduct flexibly in direct response to her final end. $A$ can only exert her flexible agency sequentially: first, toward the intermediate end (getting around the peak) and only when she succeeds at it, toward the final end. Getting around the peak operates as an end because $A$ needs instrumentally to guide her conduct directly toward it, treating it as a final end for the time being, i.e., as long as her enduring final end (re-joining the team) is blocked from view.

For any sufficiently extended pursuit, the geography of the agent's circumstances forces many segmentations of this sort. Oftentimes, we have only a rough sense of the landscape and an obstructed view of the exact location of the final end (which might be vague or moving) and of many of the intermediate places that we would need to traverse to get there. We thus need, first, 
to draft a general plan with various intermediate ends, and second, to set out to achieve these ends sequentially, exercising flexible transparent agency only within each segment. In this case, both preparing and implementing the plan might be difficult, but navigating through the segments is structurally straightforward: we just need to pursue them in the right sequence.

Segmentation and the associated fluidity get more complicated when the segments are not simply forced on us by geography but also by the scarcity of deliberative resources - that is, limitations in our ability to gather, retain, and elaborate practically relevant information. Because of this scarcity, we face a trade-off. In principle, we'd like to operate with maximal flexibility, that is, to gather and elaborate as much relevant information as possible about the exact location of the final end and the details of the landscape, and continuously update our plans accordingly. But this flexibility would soon deplete scarce resources, significantly reducing our chances of eventual success.

We must then settle on some intermediate ends, so that we can devote our energy and attention to achieving them first, even if, in hindsight, these ends might turn out to be less than optimal and, sometimes, even detrimental to final success. To settle for them amounts to focusing primarily if not exclusively on a smaller portion of our endeavors. This we achieve by imposing a temporary 'opacity,' if not even blindness, to much of the larger contexts of our present conduct, including its final end, and any other concurrent enduring ends. This opacity would then allow us to make a better (although risky) use of our scarce resources, both in deliberation and implementation.

We deliberately set restrictions on how much information we should try to gather and on how much time we should spend on the recalculation and reconfiguration of our plans. So, in 
addition to the segments that are forced on us by the geography, we need to create additional relatively rigid segments for the temporal management of our scarce resources.

\section{Absorption and Opacity}

The opacity is possible because of what Nguyen calls 'absorption': 'our capacity for submersion - for losing ourselves in a temporary agency, and momentarily blotting out our connection with our enduring values and ends' (2020a, 53). To submerge oneself in a new end is to 'phenomenally make that temporary end dominant in my reasoning, my motivation, and my practical consciousness' (2020a, 53).

As Nguyen compelling shows, striving games offer a stark illustration of this phenomenon (henceforth, unless otherwise noted, I will use 'games' to refer to 'striving games'). In games, the absorption takes a particularly strong form (also on account of their characteristic 'motivational inversion' discussed in the last section). But to reinforce Nguyen's argument against a possible skepticism about this submersion, I will point out that this capacity is central to our agency but for the simplest scenarios of full transparency.

Absorption goes beyond the mere management of attention on the task at hand. At its basis lies a structural change induced by the opacity to the more enduring ends. The opacity makes some intermediate steps acquire a temporary 'finality': they come to guide our conduct as temporarily fixed ends (this is ultimately the same phenomenon as the 'framing' role of intentions in Bratman's [1987] account of planning agency). The absorption depends on a structural discontinuity in the role of intermediate steps, which turn from mere means (within fully transparent agency) into intermediate ends (within layered agency).

Despite the discontinuity, the insulation of intermediate ends need not be absolute. Minimally, there are circuit-breakers: one immediately snaps out of the submersion and 
abandons the intermediate end in response to an emergency, such as learning that the enduring end has become impossible to reach or that some other more urgent and important task needs to be attended to. And more moderate opacity allows for the temporal suspension of the pursuit of an intermediate end while trying to gather more information about the final end and the role of the current segment in the overall plan. In some cases, one might even continue to pursue the intermediate end while diverting some resources to a possible reconsideration. There is a wide range of levels of absorption, with different degrees of permeability of the veil of opacity. But once a segment is created, a veil is imposed and by default one is supposed to ignore what lies behind it.

I wrote 'supposed to' because the temptation to lift the veil might at times be quite strong. After all, the ideal of maximally flexible agency calls for transparency. Thus, agents like us, with scarce deliberative resources, face a double challenge: the need to set up segments but also to try to stay inside them.

We manage this challenge by trying to be 'fluid': to be proficient in setting up the segments and navigating between them by correctly responding to their degrees of permeability. Central to this fluidity is the capacity appropriately to switch between staying inside a segment and getting out of it, whether temporarily or permanently. Fluidity requires some suppleness, but it is not to be confused with full flexibility. Flexibility is the proficiency of flat and fully transparent agency; fluidity is the proficiency of layered and partially opaque agency.

Failures of fluidity, as Nguyen $(2020 a, 56)$ remarks, can be seen in some faulty engagements in striving games: on the one hand, the stubbornness of those who 'get stuck' in a 
game when it is or should be over, on the other hand, the 'diffidence' of those who are unable to properly care or commit to a game when they should.

\section{Vertical Strata}

The segmentation I illustrated above is horizontal: it takes place within the pursuit of a single final end at the same level of 'resolution.' But in our agency, there is also plenty of vertical segmentation.

Return to $A$ 's scenario. The initial segmentation was in terms of a big landmark: getting around a peak. As $A$ embarks in the pursuit of this intermediate end, she also faces pressures for a more fine-grained layering: the salt flat lies behind the peak and for the time being, $A$ needs to contend with more a rugged landscape while managing her scarce resources. For instance, she might have only a partial view of how to get around the peak, so she settles on following a particular trail in the hope it might put her in a better position to figure out how best to get around the peak. And having settled on the trail, she is now concerned about the even more specific issue of the exact steps she is to take because of the slippery ground. So, to keep her balance, she needs to settle on a specific step and organize her next one accordingly.

The 'settling' in any of the more specific plans is still a matter of establishing a temporary intermediate end and be absorbed in it at the appropriate temporal resolution, that is, holding the end fixed by default for a certain amount of time, without re-calculating its appropriateness as one moves along, unlike what one would do in fully transparent agency.

These more fine-grained layers, let's call them 'strata,' are vertically nested. They are structurally analogous to segments; in that, they also depend on partial opacity and absorption. But there are also two important differences: First, strata are the product of a vertical and hierarchical articulation of a given pursuit, segments are the product of a horizontal and 
sequential one. Second, horizontal segmentation usually comes as a complete partition of a pursuit in its main stages. But each segment, in turn, often calls for more fine-grained strata. These strata, however, need not necessarily comprise the entire segment. Oftentimes, the agent might make progress in a pursuit by temporarily zooming in on quite specific steps at a more fine-grained level and then by zooming back at a more coarse-grained level, rather than having always to proceed over concatenated segments at the same level.

The degrees of resolution often correspond to different levels of opacity and absorption. At the lower level, high-resolution strata tend to have a much shorter duration. And from the lower level, the higher and more coarse-grained layers might remain more visible and accessible. Unlike the blackout curtain that might separate one segment from another, a higher stratum might appear, in a sort of bokeh effect, as the unfocused background of the lower stratum that is at the current center of the agent's attention. Fluidity is thus the capacity to establish and navigate layers of agency both horizontally and vertically.

Nguyen's main focus is on the layering and absorption required to enter and engage in games. But his account, as I am trying to show in this paper, makes us better appreciate the structural complexity of our agency in general. Not only do games illustrate what a layer is but they can also recapitulate the complexity from within. Players might face challenges that call for a combination of internal segmentation and stratification. Such layering might be made explicit in a game, be 'teleologically crisper' in Nguyen's words (2020a, 68), by such features as the institution of clear turns, the restriction of available information about future developments, the imposition of scarcity on the players, etc. Besides, games might invite players at different moments to zoom in on specific tasks or zoom out to assess their overall strategy. In sufficiently complicated games, a player might be absorbed not just in the game in general but also in a 
particular layer of that game. So, we can learn about the fluidity of agency not just by entering or exiting a game, but also in the unfolding of the game itself. This is another instance where the configuration of a game can be said to 'formalize' and 'make crisper' (to use Nguyen's expressions) the structure of agency at large.

\section{Plural Ends and Leisure Niches}

So far, I have discussed layering internal to a single final end. But there are often cases in which we are after a multiplicity of final ends (or at least under a multiplicity of potentially conflicting intermediate ends). The coordination of these ends often benefits from the layering of the individual pursuits. It allows for the temporary isolation of a single pursuit, which can be engaged single-mindedly to avoid any undue interference with other potentially conflicting goals. The same mechanism of absorption is at work here, even if what is put being the veil might be other ends rather than just other segments or strata of the current pursuit. (This coordination of plural ends by layering is, for instance, encouraged by games whose scoring system allows for independent ways of winning and neatly separates the different kinds of moves conducive to the various possible winning strategies.)

Several distinct and potentially conflicting pursuits might thus be carried out by a kind of interleaving between distinct segments and strata, that is, by taking turns between separate stints of single-minded absorption across different pursuits (as opposed to a genuine contemporaneous multi-tasking).

The interleaving does not have to take place at exactly the same level of resolution. Different pursuits might take different 'temporal textures,' activities might proceed at different paces and fill time differently, some might require continuous attention (car racing, say), others might proceed in a more desultory way (baking a cake, say), some might require some precise 
timing and cannot tolerate any delay and interruption, whereas others can be quite flexible, etc. The difference in texture allows for some activities to interleave by inserting themselves in the temporary interstices left open by slower, more delay tolerant, or higher-resolution pursuits. Some activities might just coordinate by fitting into the nooks and crannies (Nguyen 2020a, 62) left unoccupied by some other pursuits. (For instance, a game of snail-mail correspondence chess might proceed uninterrupted and without any delay at its pace and level of resolution but still leave plenty of interstices for other concurrent pursuits, given that playing that game does not require constant and continuous absorption in the interval when a move gets transmitted from one player to the other.)

An interesting feature of the interplay among the different textures of our pursuits is that, under sufficiently favorable conditions, we can often make room for some relatively long periods that are devoid of urgent demands. In these special 'leisure niches,' we can put most of our pursuits on the back burner or a temporary pause, without making irreparable damage to their prospects of eventual success.

Many of these leisure niches are the natural home for games. For they allow, within their confines, for the acquisition and pursuit of temporary ends and the strong form of absorption characteristically required by games. The isolation afforded by leisure niches makes it possible to explore the pursuit of kinds of ends that might not even be available to the agents outside of those niches. These might even include goals that, outside of that niche, would conflict with other enduring ends, or dangerous, or even morally questionable.

Leisure niches make possible, through games, the sandboxing of ends, including some potentially problematic ones. The sandboxing protects the agent's enduring ends, but it also allows for exploration and discovery. It could serve as a training ground or an innovation lab, 
where we can familiarize ourselves with what Nguyen calls the 'libraries of agency' provided by games, some of which we might then export and apply outside of the sandbox.

The sandboxing also promotes a dynamic integration of our complex agency (see Nguyen 2020a, 61-2). Rather than abandoning or banning certain potentially disruptive ends, we can pursue and explore them in earnest but only within some clearly confined and sealed spaces. But isn't this a kind of compartmentalization that divides the agent? This would be a legitimate worry if we were talking about the persistent and utter isolation of different pursuits at a higher level. That separation would likely amount to a problematic split in the agent. But the sandboxing of games stands along a continuum of the kind of dynamic integration that is achieved by the partial, temporary, and relative isolation of layering, where the different segments, strata, and niches are also kept together by our fluidity. It is no accident that we speak of the navigation between the various layers as 'fluid.' This is to indicate that this navigation is usually a smooth and seamless affair rather than a jerky and bumpy one, even if it requires moving over and zooming in and out of a rugged landscape, with views that can be partially blocked, veiled, or out of focus.

\section{Motivational Inversion}

Striving games exemplify one more important structural complexity in our agency, what Nguyen (2020a, 28) calls the 'motivational inversion' in striving play. In striving play, we adopt the ingame ends for the primary purpose of engaging in that very pursuit -i.e., for the sake of the striving. Achieving in-game ends is only a means to the striving. However, we can succeed at striving only by being fully absorbed in the game, that is, by (temporarily) embracing the ingame ends as our primary goal. It is only by immersing ourselves in the pursuit of the in-game ends that we can pursue the primary but self-effacing purpose of enjoying the striving toward the 
in-game ends. This is the motivational inversion.

As Nguyen (2020a, 55) correctly remarks, self-effacing ends are a common occurrence in our lives. Striving games merely formalize the technique of self-manipulation of our interests that makes it possible to pursue self-effacing ends. But it is also true that the motivational inversion of self-effacing ends comes with an air of paradox that might explain a possible resistance to Nguyen's account of striving play.

The main trouble with the motivational inversion seems its manipulative character: the need to induce the 'effacing' of the primary purpose (the striving) by pushing it away from our attention so that one can be fully absorbed in the pursuit of in-game ends. Why couldn't one keep both ends equally in view at the same time? The concern is that the realization that one's primary goal is just the striving might undermine the guiding role of the in-game ends since the latter ends turn out to be just means to the end of striving.

The absorption required by the inversion cannot be the source of the problem. In ordinary cases, even if the absorption in an intermediate end temporarily blocks the view of the further enduring end, there is no effacement of the latter. This is because the absorption is still part of the same instrumental order.

This can be shown by considering what happens if an agent is suddenly granted a view of the enduring ends at no cost to them. The presumption is that this view would not destabilize the intermediate end but rather confirm its role in promoting the enduring ends. Ditto for layers used for coordination across multiple ends. In principle, a temporary lifting of the veil that demarcates a layer should reveal that one's temporary engagement in that layer is compatible with the pursuit of all other enduring ends. 
This is only a presumption, however. There is no guarantee that the intermediate ends be left unscathed by a larger view of one's practical circumstances. Lifting the veil might show that an intermediate end should be immediately abandoned because it fails to contribute to or is in conflict with any of one's enduring ends. Even so, by default, we pursue an intermediate end under the defeasible assumption that it would still be upheld if we were temporarily to step outside of that layer and assess how our pursuit of that intermediate end fits within our practical predicament at large.

This is the kind of absorption required to engage in a game in the first place. If one wants to occupy a leisure niche, one can do so by embracing the temporary end of playing a certain game and thereby engaging in this temporary single-minded pursuit, against the background of the temporary receding of all the other pursuits.

Notice that this is not yet the absorption specific to the internal operation of striving games. What I am describing here is only the absorption of the standard sort, the one required to have any intermediate end so as to engage in any separate layer of agency. This kind of absorption would be required to fill a leisure niche, for instance, whether with a pastime, a craft project, or a striving game.

But once we take up a striving game, we thereby need an additional kind of absorption, the absorption in the in-game ends. This submersion is internal to the game: it concerns the proper functioning of the game as a striving game, not the role of that particular game within one's practical predicament at large. I will call this the 'in-game absorption' or 'i-absorption' for short. (To see the difference between the two absorptions, consider the agents as they are setting up or putting away a board game. At those times they are absorbed in the activity of filling the 
niche with that game, but they are not yet i-absorbed in the in-game ends, since the game has not yet started.)

What I call i-absorption is necessary to secure the proper engagement in the pursuit of the in-game ends as required by a striving game, that is, to secure that one is indeed 'striving' in trying to achieve them. While one is playing, to ascertain whether one is succeeding at the primary purpose of the game — at the striving — one has to take a half-step away from the game: one must temporarily emerge from the i-absorption and determine whether the players are experiencing the desired level and kind of engagement in the game. This is only a half-step away because, in taking it, one is not concerned with the fully external question of whether that game fits with one's enduring ends.

The difference between the two questions might not be immediately apparent because, if one finds out that the game is failing at its primary constitutive purpose (fails to bring out the desired striving), that usually shows that the game is also failing at properly filling the leisure niche. When so, one is normally expected to abandon the game, that is, to give up the end of playing that particular game. But the reserve might not be true. One might have to terminate a game (i.e., abandon the end of playing that game) even while one is experiencing the most rewarding striving thanks to one's full i-absorption in the in-end games. This might happen, for instance, if the leisure niche is suddenly shattered by the news of some serious emergency. In this case, there is no internal failure in the operation of the game, but the game still needs to be urgently abandoned.

When an emergency pierces through the game layer, hence, it goes through two different levels of isolation, the i-absorption in the in-game ends and the absorption in the project of 
filling the leisure niche by playing that game. One can break through the two absorptions at the same time but they are still two functionally distinct barriers.

Let's now return to the i-absorption. When one temporarily suspends it to check whether the game is meeting its constitutive purpose - whether the players are experiencing the intended striving — one is only half-distancing from the game. For one is not thereby giving up the end of playing that game, one is only checking on the progress of that very end.

Nonetheless, this half-step is dangerous for players who do not fully appreciate the motivational inversion of striving games. They might respond to the realization of the primacy of the purpose of striving by failing to appropriately re-engage with the in-game ends. They correctly see that the pursuit of the in-game ends is only instrumental to the striving but they fail to understand (and thereby resist) the need for the effacement of the primary purpose.

This is not to say that reflection on the structure of striving games makes engagement in them impossible. But it is true that, at first glance, there seems to be something odd if not even paradoxical about this structure. To avoid this reaction, a reflective player needs to understand that a striving game comes as a special package of ends, and it has to be embraced as such.

The game is supposed to provide the proper level of striving by matching the player's abilities and skills and by engaging them in ways that demand absorption into the in-game ends and the effacing of the striving end. A successful game reaches an equilibrium between the instrumentally secondary but phenomenologically and motivationally primary pursuit of in-game ends and the instrumentally primary but phenomenologically and motivationally secondary pursuit of the end of striving. When the motivational structure of striving games is properly understood, engagement in them can thus be stable under rational reflection. 
In sum, striving games show us that there is a further kind of layer in our agency, the one associated with the motivational inversion in the pursuit of self-effacing ends. This additional level of complexity then calls for an additional kind of fluidity, the one required to move from the pursuit of the package (via the i-absorption) to the reflective but not fully external view of the package - a view required to comprehend the structure of the package and to assess its adequacy to satisfy the self-effacing ends.

The motivational inversion is not exclusive to striving games. It is to be found in all endeavors where the agent values non-instrumentally the process of being engaged in those endeavors. That is, where the agent values the pursuit of the endeavors' ends at least in part independently of her valuing the mere achievement of those ends. Some examples are those activities, such as social tango or cooking, that are the possible objects of 'process aesthetic' appreciation and might come to be shaped by what Nguyen's (2020b) calls 'process art.'

Engagement in these endeavors for the value of the process they make possible might require acquiring or constraining the constitutive ends of the endeavors (the in-endeavor ends) at least in part because they are means to the end of experiencing that process (the process-end). Similarly to games, these endeavors would require a package of ends, in which the actual pursuit of the process-end might depend on the effacement of this end while the agent is i-absorbed in the in-endeavors ends. I say 'might' because it is an empirical question whether these endeavors require as much i-absorption as striving games. It is also plausible that, unlike the case of striving games, the achievement of the in-endeavor ends might have value independent of and prior to the mere pursuit of them (i.e., by default, one is likely to value the food one cooks, regardless of the experience of cooking or even in spite of it). 
But the basic structure of the motivational inversion exemplified by striving games appears to be present in these endeavors as well—endeavors that might enjoy a pervasive presence in our lives. This shows that striving games offer, once again, a clear model of an intricate structure of our agency and bring it to our reflective attention. This is one more demonstration of how Nguyen's insightful book helps us gain a deeper understanding of the many complexities of our fluid and layered agency.

\section{References}

Bratman, Michael. 1987. Intention, Plans, and Practical Reason. Cambridge, Mass.: Harvard University Press.

Nguyen, C. Thi. 2020a. Games: The Art of Agency. New York: Oxford University Press.

Nguyen, C. Thi. 2020b. “The Arts of Action.” Philosophers' Imprint 20 (14): 1-27. 